\title{
La cyto-aspiration prostatique transrectale
}

\author{
Eric PIATON
}

Laboratoire de Cytologie Clinique, Faculté de Médecine, Lyon Grange-Blanche, 8, avenue Rockefeller, 69373 Lyon Cedex 08, et Service d'Urologie, Hôpital de l'Antiquaille, 69321 Lyon Cedex 05.

\section{RESUME}

La cyto-aspiration prostatique transrectale à l'aiguille fine est une méthode simple, dépourvue de complications et reproductible. La sensibilité de la cytoaspiration dans le diagnostic de carcinome prostatique est de 70 à $95 \%$, et sa spécificité de 65 à $95 \%$, par rapport à la méthode biopsique. La corrélation des grades cytologique et de Gleason est de 75\%. Les complications liées à la cytoaspiration transrectale sont très faibles, à condition de prescrire une antibiothérapie prophylactique. Les indications de la cyto-aspiration prostatique sont limitées, le diagnostic formel et le grading étant apportés par l'histopathologie. Les biopsies guidées par échographie sont effectuées dans l'étape diagnostique initiale et de stadification des cancers de la prostate. La cyto-aspiration devrait plutôt être utilisée pour le diagnostic précoce, de façon systématique chez les patients consultant en urologie.

Mots-clés : prostate, cancer, cytologie, diagnostic

\section{INTRODUCTION}

La cyto-aspiration prostatique transrectale a été décrite dès 1930 par FERGUSON [5] comme une méthode diagnostique simple des cancers de la prostate, et a été particulièrement développée par EsPosTI et FRANZEN [4] au Karolinska Institute de Stockholm. Son champ d'application est pourtant resté limité, aussi bien en France qu'aux Etats-Unis, bien que sa valeur prédictive positive ait été démontrée dans de nombreuses études. Cette limitation a surtout été causée par des problèmes techniques de prélèvement qui ont entrainé de nombreux examens ininterprétables ou faussement négatifs dans les équipes insuffisamment expérimentées. La place de la cyto-aspiration prostatique transrectale par rapport aux biopsies est controversée. Actuellement, on devrait considérer la cyto-aspiration comme un outil de dépistage orienté à effectuer en milieu urologique, et non comme une méthode remplaçant ou complétant les biopsies effectuées dans le diagnostic initial ou dans l'indispensable étape de stadification préopératoire des cancers de la prostate.

\section{LA TECHNIQUE DE PONCTION}

La cyto-aspiration prostatique peut être réalisée en ambulatoire, sans anesthésie préalable ni préparation rectale, et sans bilan biologique particulier. Le dosage du PSA et le toucher rectal doivent être effectués avant la ponction, et la prise d'un antibiotique (Péflacine $400 \mathrm{mg}$ la veille au soir et le matin de la consultation, ou Norfloxine $400 \mathrm{mg} 1 / 2$ heure avant le prélèvement et 2 fois/jour pendant 5 jours) est souhaitable.

Le prélèvement est réalisé sur un patient en décubitus dorsal, jambes repliées. Un robinet à 3 voies est vissé sur le corps d'une seringue de $50 \mathrm{ml}$ dans le piston de laquelle 
est disposé un taquet auto-bloquant (Figure 1). Une aiguille de 22 gauge de $9 \mathrm{~cm}$ (aiguille à PL) est adaptée sur le robinet. Il est très utile d'aspirer $1 \mathrm{ml}$ de fixateur directement dans le corps de la seringue (Polyéthylène-Glycol 1500 Merck $^{\circledR}$ en solution à $2 \%$ dans l'éthanol à $50^{\circ}$, conditionné dans un tube à hémolyse de $5 \mathrm{ml}$ ). Le robinet à 3 voies est ensuite bloqué. Le piston est alors tiré à fond et le taquet s'enclenche, créant une dépression importante dans le corps de la seringue. Deux gants sont enfilés l'un sur l'autre (main effectuant le toucher rectal et le prélèvement) et l'aiguille est glissée entre les gants jusqu'à la pulpe de l'index, les autres doigts maintenant la seringue. L'index comprime la prostate (c'est l'étirement de la capsule avant son franchissement qui est douloureux), et l'aiguille est poussée de 1 à $3 \mathrm{~cm}$ dans le parenchyme. Après prise des repères on peut effectuer 6 à 8 ponctions sans ressortir le doigt. S'il existe une zone suspecte, elle peut être ponctionnée 2 à 3 fois, avec prélèvements dans le lobe contro-latéral. L'aiguille est animée de mouvements

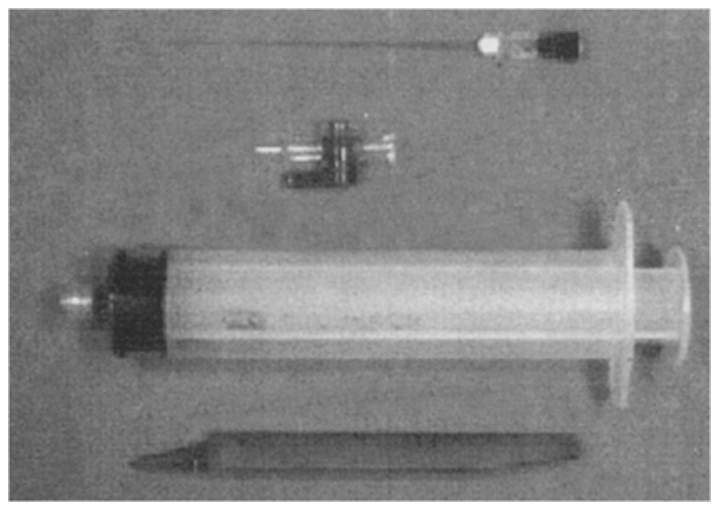

Figure 1 : Le matériel de cyto-aspiration : noter l'aiguille spinale de 22 gauge de $9 \mathrm{~cm}$, le robinet adaptable, la seringue de $50 \mathrm{ml}$, le taquet auto-bloquant à insérer dans le corps de la seringue. d'avancée et de recul pour améliorer la collecte cellulaire. Entre chaque ponction le robinet est refermé. Lorsque la cyto-aspiration est terminée, l'aiguille est retirée et le reste du fixateur est aspiré dans la seringue. La suspension cellulaire est alors réinjectée dans le tube à hémolyse et adressée par la poste au laboratoire spécialisé dans une enveloppe matelassée. L'envoi doit être accompagné de renseignements cliniques (taux de PSA, toucher rectal et aspects échographiques, mode de prélèvement : ponction systématique bilatérale, ponction sur un nodule suspect (Figure 2).

La préparation et la réalisation de la cytoaspiration transrectale n'excèdent pas 5 minutes, à condition de ne pas faire le prélèvement sous échographie. Ce dernier procédé nécessite au moins $1 / 4$ d'heure et l'utilisation d'une aiguille de $17 \mathrm{~cm}$, de même qu'une technique plus sophistiquée. On peut bien entendu faire la cyto-aspiration transrectale en complément d'une exploration biopsique échoguidée, ce qui est toutefois d'un intérêt limité (voir le paragraphe "indications").

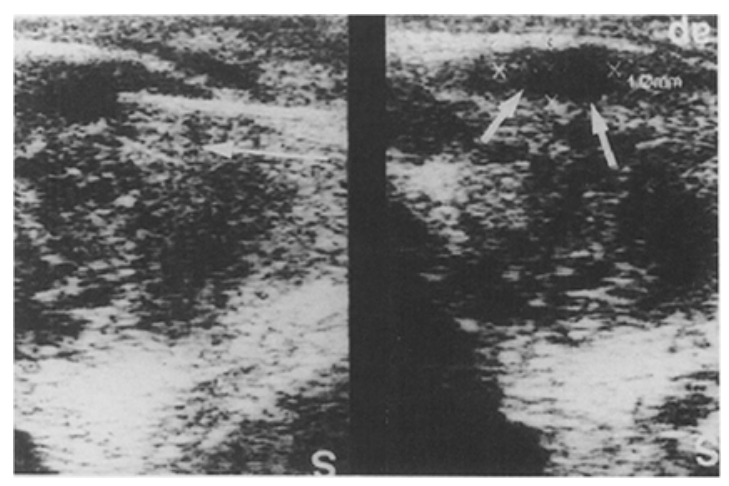

Figure 2 : Aspect échographique d'un nodule hypoéchogène de $10 \mathrm{~mm}$ de grand axe de la zone périphérique postérieure de la prostate. L'aiguille traverse la capsule et atteint le nodule (à gauche). 


\section{L'INTERPRETATION CYTOLOGIQUE (VALEUR ET LIMITES)}

L'examen cytopathologique est réalisé sur des étalements colorés selon la méthode de Papanicolaou, et la réponse peut être donnée en 24 à 48 heures. Après indication de la qualité du matériel obtenu (cellularité, conservation, sang) les éléments cellulaires sont observés à fort grossissement et décrits : l'épithélium prostatique normal ou hyperplasique est uni ou pluristratifié, et des cellules basales sont visibles. Les noyaux sont ronds ou ovalaires, non nucléolés, séparés par des espaces cytoplasmiques et des limites cellulaires dessinant des aspects en rayon de miel. On peut observer des éléments conjonctivo-musculaires lisses de soutien et des corps amylacés d'origine intracanalaire ou kystique. Les aspects adénocarcinomateux sont à la fois architecturaux et cyto-nucléaires : on observe une perte de cohésion intercellulaire aboutissant dans le carcinome peu différencié à de nombreux noyaux nus nucléolés (Figure 3 ). Les noyaux sont globalement augmentés de volume, irréguliers et porteurs de nucléoles très hypertrophiés, sauf dans certains carcinomes indifférenciés de la prostate.

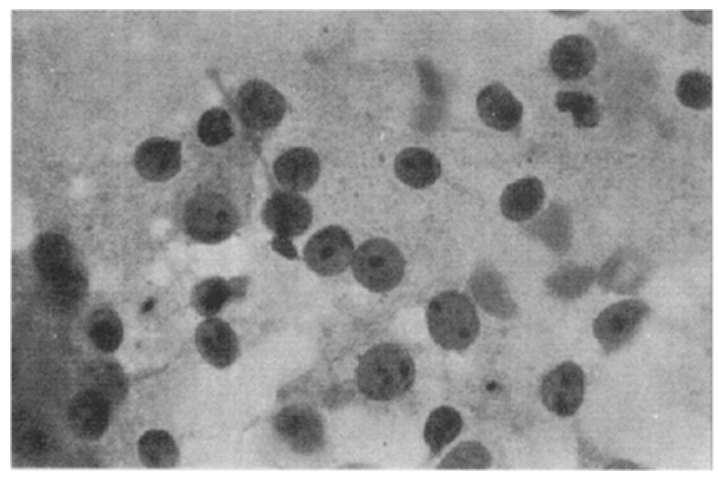

Figure 3 : Aspect cytologique à fort grossissement d'un adénocarcinome peu différencié (grade III) Les noyaux très nucléolés. La désorganisation architecturale est totale.
La conclusion cytopathologique est concise : "il n'a pas été vu d'éléments carcinomateux au niveau du matériel examiné", ou bien "aspect cytologique compatible avec une hyperplasie adéno-myomateuse bénigne" lorsque les éléments épithéliaux et conjontivo-musculaires bénins sont très abondants. Les prostatites subaigües ou granulomateuses (diagnostic différentiel du carcinome parfois) sont particulièrement bien mises en évidence. Dans le diagnostic de malignité [1-3], la concordance avec les résultats histopathologiques peut être évaluée à : 1) sensibilité : 70 à $95 \%$ (91\% en moyenne), 2$)$ spécificité : 65 à $95 \%$ ( $88 \%$ en moyenne). En fait des résultats inférieurs, surtout en terme de sensibilité doivent être attendus si la prévalence du carcinome est faible dans la population étudiée. Si l'on s'intéresse à la cyto-aspiration prostatique comme méthode complémentaire du toucher rectal et de l'échographie, il faut savoir que seuls les résultats positifs auront une valeur, et qu'il faudra interpréter ceux-ci en fonction du grade. La cyto-aspiration transrectale échoguidée peut également être utilisée pour obtenir un diagnostic et un grade lors des reprises évolutives locales après prostatectomie $(25 \%$ à $30 \%$ des cancers de prostate opérés).

Les éléments carcinomateux peuvent être gradés de I à III, ce qui permet une corrélation assez satisfaisante avec le grade de Gleason dans les formes moyennement et peu différenciées. Dans notre expérience, tous les résultats cytologiques positifs de grade II et III sont des cancers histologiquement démontrés. Les grades I peuvent correspondre à des carcinomes ou à des lésions de dysplasie intra-canalaire [7]. La corrélation globale moyenne des grades cytologique et biopsique est de 75\% [2], avec une sousestimation fréquente du grade par la cytologie $[3,6,8]$. Les grades II et III sont toujours corrélés avec des biopsies comportant des zones de grade 4 de Gleason dans $50 \%$ et $70 \%$ des cas, et de grade 5 de Gleason dans $10 \%$ et $18 \%$ des cas respectivement [7]. 


\section{LES INDICATIONS}

On a pu dire encore récemment que la cytoaspiration prostatique reconnaissait les mêmes indications formelles que les biopsies, c'est à dire les anomalies palpables au toucher rectal. Cette position ne peut plus être défendue, car on dispose de moyens échographiques permettant de prélever de façon dirigée les anomalies non palpables de la glande prostatique et de stadifier de façon systématique et sérieuse les carcinomes. De plus, les systèmes modernes de biopsies automatiques (Biopty ${ }^{\circledR}$ ) permettent d'obtenir un diagnostic histopathologique formel et un grade selon Gleason avec un taux de complications remarquablement faible. Il ne faut donc pas utiliser la cytoaspiration de façon exclusive ou concurrentielle des biopsies, ni même de façon complémentaire lors d'une exploration échographique. On peut toutefois admettre une exception lorsqu'on désire réaliser des corrélations cyto-biopsiques dans un but de mise au point et d'apprentissage au sein d'une nouvelle collaboration urologue-cytopathologiste. La cyto-aspiration prostatique n'est pas non plus une méthode fiable de dépistage, au sens médico-économique.

Selon nous, les indications réellement utiles des cyto-aspirations prostatiques sont les tentatives de diagnostic précoce des cancers de la prostate chez les patients vus en consultation d'urologie. Cette proposition est ambitieuse : elle suppose que l'examen cytopatho-logique apporte des informations complémentaires à la pratique combinée du toucher rectal, de l'échographie et du dosage systématique du PSA. Il faut savoir que la valeur prédictive positive de ces trois examens, utilisés de façon combinée (TR suspect, zone hypoéchogène postérieure, PSA $>4 \mathrm{ng} / \mathrm{ml}$ ) n'est pas supérieure à $65 \%$. De plus, cette démarche suppose que tous les patients vus en consultation d'urologie pourront bénéficier d'une cyto-aspiration, et que l'urologue admette que la sensibilité imparfaite de la méthode sera compensée par un diagnostic carcinologique de quasicertitude dans les grades II et III.
Les propositions formulées ci-dessus n'empêchent nullement de pratiquer des prélèvements sur les anomalies palpables et/ou échographiques de la prostate. Le diagnostic des récidives locales après prostatectomie radicale, par des prélèvements échoguidés, est également possible. Toutefois la méthode de référence, permettant un diagnostic formel et un grading tumoral selon Gleason reste la biopsie échoguidée.

Des indications plus particulières sont l'étude de l'ADN tumoral par cytométrie en flux à partir des suspensions cellulaires obtenues. En effet la cyto-aspiration prostatique réalise une dissociation mécanique favorisant l'obtention de noyaux nus. Le matériel cyto-aspiré peut voyager par voie postale jusqu'au laboratoire réalisant l'analyse de l'ADN en flux. Précisons qu'il est indispensable de réaliser un contrôle cytopathologique conventionnel des suspensions cellulaires dont on étudie l'ADN tumoral.

\section{LES COMPLICATIONS}

Les complications (épididymite, hématurie transitoire, hémospermie, infection) sont très rares, estimées à $0,4 \%$ par BENSON [2]. Dans notre expérience, une fièvre supérieure à $38^{\circ}$ a été notée chez $4 \%$ des patients ayant débuté l'antibiothérapie après le prélèvement. Par conséquent on peut dire qu'il n'existe pas de véritable contre-indication à la cyto-aspiration prostatique, à condition de prescrire une antibiothérapie de protection.

\section{LE COUT}

La ponction prostatique (cytologique ou biopsique non échoguidée) est cotée K 20 soit 248 francs ( $K=12,40$ francs). L'échographie prostatique, quant à elle, est cotée K 35 soit 434 francs. La ponction prostatique (cytologique ou biopsique) échoguidée est donc cotée $K 35+K 20 / 2$ soit 558 francs.

La technique et l'interprétation cytologique sont cotées BP 120 soit 211,20 F (BP $=1,76$ 
F). Au total, la facture pour le patient sera de 769,20 francs ou de 459,20 francs selon que le prélèvement a été ou n'a pas été échoguidé.

\section{REFERENCES}

1. BALLENTINE CARTER H, RIEHLE RA, KOIZUMI JH, AMBERSON J, DARRACOTT VAUGHAN E. : Fine-needle aspiration of the abnormal prostate : a cytohistological correlation. J. Urol., 1986, 135 : 294-298.

2. BENSON MC. : Fine-needle aspiration of the prostate. NCI Monographs, 1988, $7:$ 19-24.

3. BROMBERG WD, GRAHAM JB. : Prostatic needle aspiration biopsy. Advances in Urology, 1989, 2 : $25-40$.

4. ESPOSTI PL, FRANZEN S. Transrectal aspiration biopsy of the prostate : a re-evaluation of the method in the diagnosis of prostate carcinoma. Scand. J. Urol. Nephrol. [suppl], 1980, 55 : 49-52.

5. FERGUSON RS. : Prostatic neoplasms : their diagnosis by needle puncture and aspiration. Am. J. Surg., 1930, $9:$ 507-511.

6. HOSTETTER AL, PEDERSEN KV, GUSTAFSSON BL, MANSON JC, BOERYD BRG. : Diagnosis and localization of prostate carcinoma by fine-needle aspiration cytology and correlation with histologic whole-organ sections after radical prostatectomy. Am. J. Clin. Pathol., 1990, 94 : 693-697.

7. PIATON E. : La cytologie dans le diagnostic positif et le grading des cancers de la prostate : quelles indications reste-t-il, à l'heure des biopsies automatiques et de l'échographie endorectale ? Bull. Cancer, 1992, 79:67-80.

8. ZATTONI F, PAGANO F, REBUFFI A, COSTANTIN G. Transrectal thin-needle aspiration biopsy of prostate : four years' experience. Urology, 1988, $22: 69-72$.

\section{ABSTRACT \\ Transrectal fine-needle aspiration of the prostate}

\section{E. Piaton}

Laboratoire de Cytologie Clinique, Faculté de Médecine, Lyon Grange-Blanche, 8, avenue Rockefeller, 69373 Lyon Cedex 08, et Service d'Urologie, Hôpital de l'Antiquaille, 69321 Lyon Cedex 05.

The diagnosis of prostate adenocarcinoma by fine-needle aspiration cytolo- gy (FNAC) has taken long to accept as a simple, safe and reproducible procedure. The transrectal approach, as described for many years, remains the simplest method. Cytological samples need to be fixed with adequate solutions. Local anesthesia is not useful, and ultrasonographic guidance is not necessary. Reported sensitivity and specificity values of FNAC are 70-95\% and $65-95 \%$ respectively, when biopsy is taken as the diagnostic reference. The correlation of cytological and Gleason's grading is about $75 \%$. Complications after transrectal sampling are very low, provided previous antibiotherapy is administrated. The indications for performing FNAC are limited, a histopathological diagnosis being necessary. Ultrasonographicallyguided biopsies should be done in every diagnostic and staging attempt. FNAC should be done more systematically in order to provide early diagnosis in patients presenting with non specific urologic symptoms.

Key words : prostate carcinoma, fine-needle aspiration, diagnosis. 